\title{
Comparación fitosociológica de los bosques de Belloto (Beilschmiedia, Lauraceae) en Chile central
}

Phytosociological comparisons of Belloto (Beilschmiedia, Lauraceae) forests in central Chile

\author{
CARLOS RAMIREZ ${ }^{1}$, CRISTINA SAN MARTIN ${ }^{1}$, JOSE SAN MARTIN², \\ RODRIGO VILLASEÑOR ${ }^{3}$ \\ ${ }^{1}$ Instituto de Botánica, Universidad Austral de Chile, Casilla 567, Valdivia, Chile. \\ ${ }^{2}$ Instituto de Biología Vegetal y Biotecnología, Universidad de Talca, Casilla 747, Talca, Chile. \\ ${ }^{3}$ Laboratorio de Botánica, Facultad de Ciencias, Universidad de Playa Ancha \\ de Ciencias de la Educación, Casilla 34-V, Valparaíso, Chile.
}

\begin{abstract}
SUMMARY
Beilschmiedia miersii (Gay) Kosterm. (Belloto del Norte) and Beilschmiedia berteroana (Gay) Kosterm. (Belloto del Sur) are sclerophyllous trees of the family Lauraceae that are endemic to central Chile. They form mixed evergreen forests of azonal character and have a fragmented distribution. The Belloto del Norte forest grows in the coastal zone of the sclerophyllous forest region, while the Belloto del Sur forest grows in the pre-mountain Andean zone of the temperate deciduous forest region of central Chile. A phytosociological comparison of these forests was accomplished using a vegetation table formed by 18 vegetation samples taken in representative stands of both forests. The floristic similarity between the forests was so low that they could be differentiated into two plant associations, which was confirmed by a multivariate statistical analysis of classification and ordination. Although the Belloto del Norte Forest has a greater floristic richness, it is due to the annual alochtonous undergrowths. Woody plants and perennial weeds dominate the biological spectrum of both communities. The Belloto del Sur forest appears to be more floristically homogeneous. The name Nothofago-Beilschmiedietum berteroanae ass. nova was proposed for the sinotaxonomical classification of the Belloto del Sur forest, while the Belloto del Norte forest was named Beilschmiedietum miersii Schmith. Despite the fact that both forest associations are currently represented in the System of Wild Protected Areas by the government of Chile (SNASPE), they are endangered. Therefore, this study intends to serve as the basis for a future ecological restoration of the forests.
\end{abstract}

Key words: Beilschmiedia, sclerophyllous forest, phytosociology, central Chile.

\section{RESUMEN}

Beilschmiedia miersii (Gay) Kosterm. (Belloto del Norte) y Beilschmiedia berteroana (Gay) Kosterm. (Belloto del Sur) son árboles esclerófilos de la familia Lauraceae endémicos de Chile central, que forman bosques perennifolios mixtos de distribución fragmentada. El bosque de Belloto del Norte crece en la zona costera de la Región de los Bosques Esclerófilos y el de Belloto del Sur, en la precordillera andina de la Región de los Bosques Caducifolios Templados. Ambos presentan carácter azonal. Se realizó una comparación fitosociológica de estos bosques utilizando una tabla fitosociológica formada por 18 censos de vegetación levantados en rodales representativos de ambas asociaciones. La similitud florística entre los bosques es tan baja, que permite diferenciar dos asociaciones vegetales, confirmadas por los análisis estadísticos multivariados de clasificación y de ordenación. Aunque el bosque de Belloto del Norte tiene una mayor riqueza florística, ella se debe a la presencia de malezas anuales alóctonas. En el espectro biológico de ambas comunidades dominan plantas leñosas y hierbas perennes. El bosque de Belloto del Sur aparece como más homogéneo florísticamente. Se propone el nombre de Nothofago-Beilschmiedietum berteroanae ass. nova para el bosque de Belloto del Sur y se establece su clasificación sintaxonómica junto con la del bosque de Belloto del Norte, el Beilschmiedietum miersii Schmith. A pesar de que actualmente ambas asociaciones están representadas en las unidades del Sistema de Areas Silvestres Protegidas por el Estado de Chile (SNASPE), ellas presentan serio peligro de conservación, por lo que este estudio pretende servir de base para una futura restauración ecológica de ellas.

Palabras claves: Beilschmiedia, bosques esclerófilos, fitosociología, Chile central. 
BOSQUE 25(1): 69-85, 2004

Comparación fitosociológica de los bosques de Belloto (Beilschmiedia, Lauraceae) en Chile central

\section{INTRODUCCION}

En Chile central existen dos especies endémicas del género Beilschmiedia (Lauraceae), Beilschmiedia miersii (Gay) Kosterm. (Belloto del Norte) y Beilschmiedia berteroana (Gay) Kosterm. (Belloto del Sur) $(1,2)$. Se trata de dos árboles perennifolios esclerófilos, que ocupan los ambientes higrófilos de quebradas $(3,4)$. A pesar de sus similares requerimientos de sitio, ellos presentan áreas de distribución disyuntas (figura 1). B. miersii crece en quebradas de la cordillera de la costa entre Huanquén $\left(32^{\circ} \mathrm{S}\right)$ y Altos de Cantillana $\left(34^{\circ} \mathrm{S}\right)$, en las Regiones V y VI y Región Metropolitana (5), B. berteroana se distribuye unos 70 km más al sur, entre Sagrada Familia $\left(35^{\circ} \mathrm{S}\right)$, en la VII Región y el estero Gallipavo ( $\left.36^{\circ} 48^{\prime} \mathrm{S}\right)$ en la VIII Región (6). Ambas especies se distribuyen en forma fragmentada, ocupando los biótopos más húmedos, por la influencia de agua edáfica y la neblina costera.

Las dos especies tienen serios problemas de conservación debido a la acción antrópica que ha ido reduciendo sus hábitats (7). El Belloto del Sur ha sido declarado oficialmente en peligro de extinción, asignándosele alta prioridad en las acciones de conservación ejecutadas por la Corporación Nacional Forestal de Chile (CONAF). El Belloto del Norte, cuyas poblaciones son más abundantes, se considera vulnerable (8).

Las dos especies forman bosques fisonómica y florísticamente definidos y distintos de aquellos que los circundan $(6,9,10)$ y corresponden a asociaciones vegetales boscosas bien delimitadas, ubicadas en diferentes regiones geográficas, aunque contiguas. Los bosques de Belloto del Norte forman parte de la Región de los Bosques esclerófilos de Chile central establecida por Schmithüsen (11); específicamente pertenecen a aquellos bosques esclerófilos más húmedos incluidos en el Orden fitosociológico Cryptocaryetalia que prosperan en quebradas de la cordillera costera (12). Los Bosques del Belloto del Sur forman parte de la vegetación de la Región de los Bosques Caducifolios Templados (11) o Bosques Maulinos en sentido amplio, cuya sintaxonomía fue recientemente actualizada por Amigo et al. (13). Estos bosques se ubican en la precordillera andina de la región mencionada y avanzan hacia el Sur por la Depresión Intermedia.

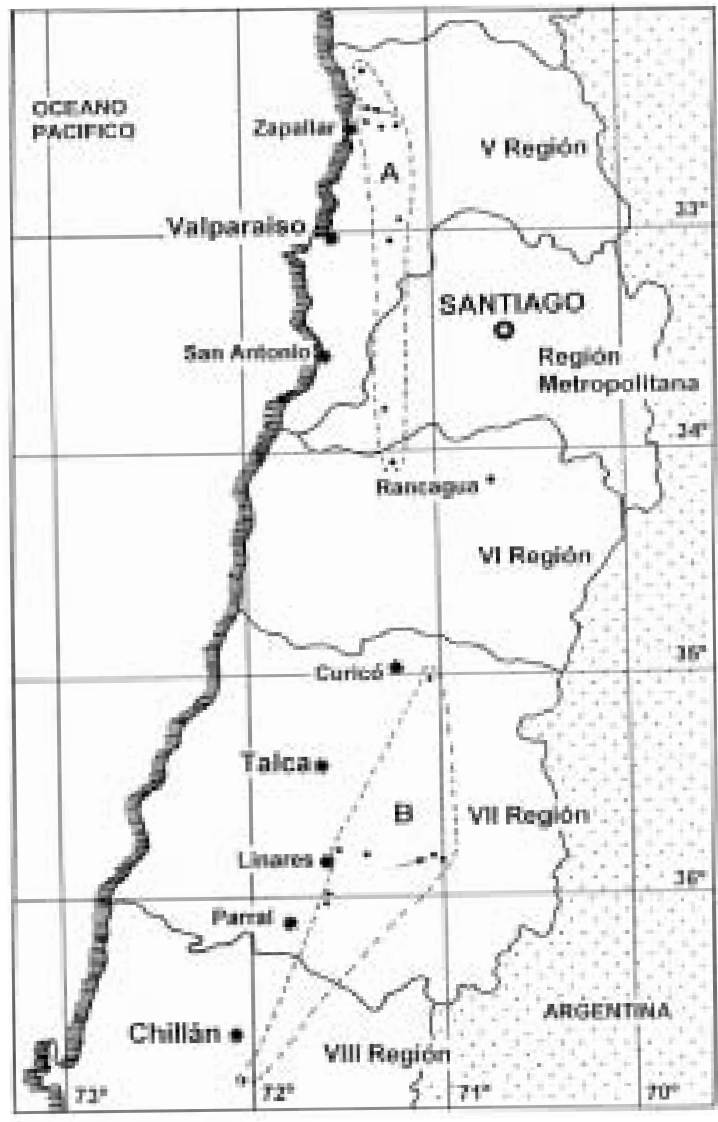

Figura 1. Chile central. Las líneas cortadas delimitan las áreas de distribución del Belloto del Norte (A) y del Belloto del Sur (B). Los puntos indican lugares de colecta citados en la literatura y las flechas los lugares de muestreo.

Central Chile. The broken lines show the distribution areas of the Belloto del Norte (A) and Belloto del Sur (B) forests. The points indicate study sites according to the literature, while the arrows show the samples sites.

El presente estudio es una comparación fitosociológica de estas dos comunidades boscosas, tendiente a delimitar su estatus fitosociológico, especialmente sintaxonómico y actualizar su nomenclatura. Además, se considera importante estudiar la estructura florística de ellos para tener antecedentes que a futuro permitan una restauración de estas asociaciones boscosas, así como establecer, en forma preliminar y mediante análisis estadísticos multivariados de las tablas de vegetación, los requerimientos de sitio de sus principales especies. 


\section{MATERIAL Y METODOS}

Area de estudio. Los sitios de estudio en el Norte del área de distribución de B. miersii fueron la Quebrada La Ceniza, ubicada al Norte de Zapallar, V Región, y en la parte central del área de distribución de $B$. berteroana, en el sector de Hornillos, en la precordillera de Linares, VI Región (figura 1).

El clima ha sido clasificado como del tipo mediterráneo (14), abarcando los bioclimas mesomediterráneo seco y mesomediterráneo húmedo (15). Las diferencias climáticas entre ambos lugares de trabajo se aprecian en los diagramas climáticos de Zapallar y Linares (figura 2). En Zapallar, ubicado en el extremo norte del área de $B$. miersii, la precipitación promedio anual alcanza a $384 \mathrm{~mm}$, mientras que en Linares, ubicado frente a la parte central del área de B. berteroana ésta sube a 1.007 $\mathrm{mm}$. Los promedios de temperatura anual son, en cambio, muy próximos entre sí, $14,2^{\circ} \mathrm{C}$ para Zapallar y $13,9^{\circ} \mathrm{C}$ para Linares. La diferencia más importante en el clima de ambos lugares la constituye la extensión del período de sequía estival que en Zapallar llega a 7 meses, mientras que en Linares se restringe a sólo 4 meses en el año. Para esta última estación meteorológica se mencionan incluso varios meses con exceso de precipitación en invierno (16).

Metodología. La base de este trabajo la constituyen 18 censos de vegetación levantados con la metodología fitosociológica de la Escuela Zürich-
Montpellier (17). En los bosques del Belloto del Norte se levantaron 8 censos a lo largo de la Quebrada La Ceniza ( $32^{\circ} 32^{\prime} \mathrm{S}$ y $71^{\circ} 24^{\prime} \mathrm{W}$ ), ubicada al norte de Zapallar (figura 1). Los 10 censos de los bosques de Belloto del Sur se levantaron en el sector Hornillos ( $35^{\circ} 52^{\prime} \mathrm{S}$ y $71^{\circ} 06^{\prime} \mathrm{W}$ ), en la ribera norte del río Ancoa, en la provincia de Linares (figura 1). Dado el pequeño tamaño de los rodales, los censos se tomaron en superficies de $100 \mathrm{~m}^{2}$. En cada uno de ellos se hizo una lista de las especies presentes y luego se determinó la cobertura-densidad de sus individuos, expresándola en porcentaje de cobertura de la parcela (18). Para coberturas bajo $1 \%$ se usaron los signos "+" y "r", el primero cuando se presentaba varios, y el segundo cuando se exponía sólo un individuo de la especie en cuestión (19).

La comparación florística se realizó sobre la base de tablas fitosociológicas separadas para cada comunidad boscosa y consideró la nomenclatura y origen de acuerdo a Marticorena y Quezada (20) y el espectro biológico, determinando las formas de vida con la clave de Mueller-Dombois y Ellenberg (21). En las tablas de cada bosque se determinó, además, un valor de importancia para sus especies de acuerdo a Wikum y Shanholtzer (22), ponderando la frecuencia y la cobertura de las especies en los censos.

La tabla fitosociológica, transformada a una matriz de datos con los valores de cobertura y subiendo los signos "+" y "r" a la unidad, fue sometida a análisis estadísticos multivariados de clasificación y ordenación, tanto de especies como
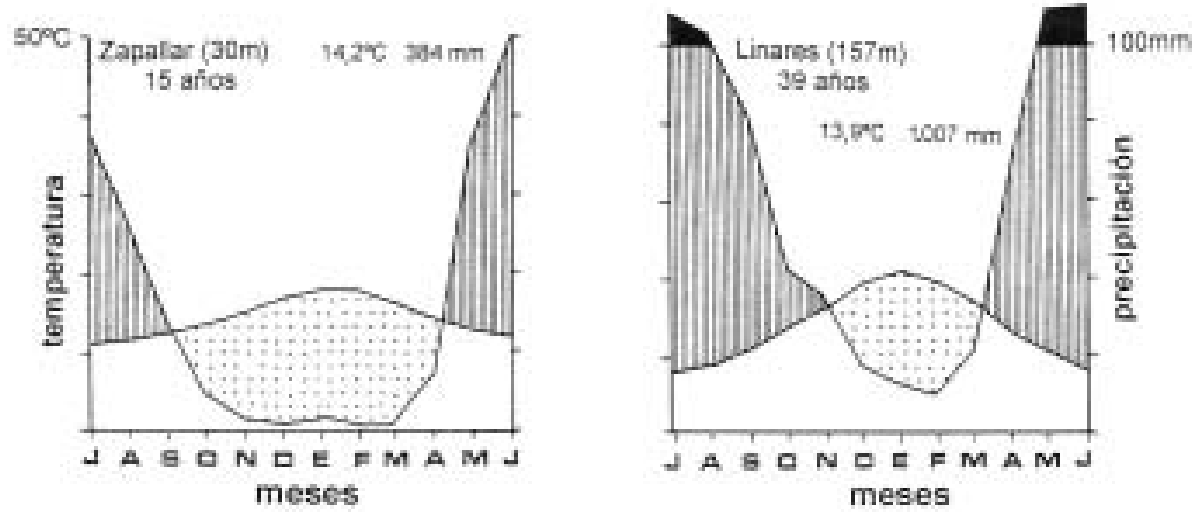

Figura 2. Diagramas climáticos de Zapallar y Linares según Hajek y Di Castri (1975).

Climatic diagram of Zapallar and Linares, after Hajek and Di Castri (1975). 
BOSQUE 25(1): 69-85, 2004

Comparación fitosociológica de los bosques de Belloto (Beilschmiedia, Lauraceae) en Chile central

también de censos (23). Se realizaron análisis de componentes principales y de conglomerados con programas estadísticos $(24,25)$. En el análisis de conglomerados se utilizó el índice de similitud de Pearson (26) para la comparación y el algoritmo del salto mínimo para construir el dendrograma (27).

La nomenclatura fitosociológica utilizada sigue al Código de Nomenclatura Fitosociológica (28).

\section{RESULTADOS}

La estructura florística de cada bosque se presenta en los cuadros 1 y 2 . Un completo catálogo de las especies vegetales presentes en estos cuadros se presenta en el Anexo, donde se entrega posición sistemática, forma de vida y origen fitogeográfico de cada una.

\section{CUADRO 1}

Estructura florística del bosque de Belloto del Norte. Floristic structure of the Belloto del Norte forest.

\begin{tabular}{|c|c|c|c|c|c|c|c|c|}
\hline Censo $\mathrm{N}^{\circ}$ & 1 & 2 & 3 & 4 & 5 & 6 & 7 & 8 \\
\hline Altitud (m s.n.m.) & 80 & 100 & 120 & 80 & 80 & 120 & 100 & 100 \\
\hline Exposición & $\mathrm{SO}$ & SO & SO & $\mathrm{O}$ & $\mathrm{O}$ & $\mathrm{SO}$ & SO & SO \\
\hline Número de especies & 31 & 30 & 26 & 30 & 21 & 18 & 15 & 30 \\
\hline Beilschmiedia miersii & 20 & 40 & 60 & 40 & 60 & 20 & 30 & 30 \\
\hline Schinus latifolia & + & 40 & 10 & 5 & . & + & 10 & + \\
\hline Stellaria media & + & + & + & + & + & + & + & . \\
\hline Cryptocarya alba & 20 & + & . & . & 20 & 10 & 40 & 10 \\
\hline Blepharocalyx cruckshanksii & 10 & + & . & . & 5 & + & + & 20 \\
\hline Lithrea caustica & 20 & + & + & + & 5 & . & . & 10 \\
\hline Peumus boldus & 10 & + & + & + & . & . & + & . \\
\hline Cestrum parqui & + & + & + & . & + & + & . & + \\
\hline Eupatorium glechonophyllum & . & + & + & + & + & . & + & + \\
\hline Adiantum chilense var & + & + & . & + & 20 & . & . & + \\
\hline Nothoscordum striatellum & . & + & + & + & + & . & . & + \\
\hline Myrceugenia exsucca & 20 & . & . & . & . & 60 & 10 & + \\
\hline Scirpus cernuus & 5 & + & . & + & . & . & . & + \\
\hline Equisetum bogotense & + & . & + & + & . & . & . & + \\
\hline Cyperus eragrostis & + & + & + & . & . & . & . & + \\
\hline Proustia pyrifolia & + & . & . & . & + & + & . & + \\
\hline Argythamnia tricuspidata & + & . & . & . & + & + & + & . \\
\hline Sicyos bryoniifolius & + & . & + & + & . & . & . & + \\
\hline Euphorbia peplus & . & + & + & + & + & . & . & . \\
\hline Azolla filiculoides & . & + & + & + & . & . & . & + \\
\hline Sanicula crassicaulis & . & & + & . & + & + & + & . \\
\hline Hydrocotyle ranunculoides & + & 20 & . & . & . & . & . & 5 \\
\hline Trevoa trinervis & . & 5 & + & + & . & . & . & . \\
\hline Rumex conglomeratus & + & + & . & + & . & . & . & . \\
\hline Lythrum album & + & + & + & . & . & . & . & . \\
\hline Azara celastrina & + & + & . & . & . & . & . & + \\
\hline Fumaria capreolata & + & . & + & . & + & . & . & . \\
\hline Maytenus boaria & . & + & + & . & . & . & . & + \\
\hline
\end{tabular}


BOSQUE 25(1): 69-85, 2004

Comparación fitosociológica de los bosques de Belloto (Beilschmiedia, Lauraceae) en Chile central

\begin{tabular}{|c|c|c|c|c|c|c|c|c|}
\hline Sonchus asper & . & + & + & . & . & . & . & + \\
\hline Ranunculus muricatus & . & + & + & + & . & . & . & . \\
\hline Luma chequen & . & . & . & 20 & . & + & . & · \\
\hline Myrceugenia obtusa & . & . & . & . & . & + & . & 10 \\
\hline Solanum maglia & . & . & . & . & 5 & + & . & . \\
\hline Urtica dioica & + & . & . & . & . & + & . & . \\
\hline Rhaphithamnus spinosus & + & . & . & . & . & . & . & + \\
\hline Lemna minuscula & + & . & . & . & . & . & . & + \\
\hline Paspalum distichum & + & . & . & + & . & . & . & . \\
\hline Lardizabala biternata & + & . & . & . & . & . & . & + \\
\hline Citronella mucronata & + & . & . & + & . & . & . & . \\
\hline Baccharis linearis & + & . & + & . & . & . & . & . \\
\hline Fuchsia lycioides & . & + & . & . & + & . & . & . \\
\hline Veronica anagallis-aquatica & . & + & . & + & . & . & . & . \\
\hline Geranium core-core & . & + & . & . & . & . & + & . \\
\hline Holcus lanatus & . & . & + & . & . & · & · & + \\
\hline Oxalis rosea & . & . & + & . & . & + & . & · \\
\hline Poa аппиа & . & . & + & + & . & . & . & . \\
\hline Plantago lanceolata & . & . & . & + & . & . & . & + \\
\hline Alstroemeria haemantha & . & . & . & + & + & . & . & · \\
\hline Verbascum virgatum & . & . & . & + & . & . & . & + \\
\hline Adenopeltis serrata & . & . & . & . & + & . & . & + \\
\hline Coniza bonariensis & + & . & . & . & . & . & . & · \\
\hline Gratiola peruviana & + & . & . & . & . & . & . & . \\
\hline Aristotelia chilensis & + & . & . & . & . & . & . & . \\
\hline Quillaja saponaria & + & . & . & . & . & . & . & . \\
\hline Calceolaria dentata & . & + & . & . & . & . & . & . \\
\hline Acacia caven & . & + & . & . & . & . & . & . \\
\hline Plantago major & . & + & . & . & . & . & . & · \\
\hline Gilliesia graminea & . & + & . & . & . & . & . & · \\
\hline Puya chilensis & . & . & + & . & . & . & . & . \\
\hline Aira caryophyllea & . & . & + & . & . & · & . & . \\
\hline Galium aparine & . & . & . & + & . & . & . & . \\
\hline Mimulus glabratus & . & . & . & + & . & . & . & - \\
\hline Senna candolleana & . & . & . & + & . & . & . & . \\
\hline Silybum marianum & . & . & . & + & . & . & . & . \\
\hline Taraxacum officinale & . & . & . & + & . & . & . & - \\
\hline Capsella bursa-pastoris & . & . & . & + & . & . & . & . \\
\hline Dioscorea bryonniifolia & . & . & . & . & + & . & . & . \\
\hline Diplolepis menziesii & . & . & . & . & + & . & . & . \\
\hline Stachys grandidentata & . & . & . & . & + & . & . & . \\
\hline Solanum nigrum & . & . & . & . & . & + & . & . \\
\hline Torilis nodosa & . & . & . & . & . & + & . & . \\
\hline Myrceugania ovata & . & . & . & . & . & + & · & · \\
\hline Cissus striata & . & . & . & . & . & . & + & . \\
\hline Euphorbia portulacoides & . & . & . & . & . & . & + & . \\
\hline Gamochaeta americana & . & . & . & . & . & . & + & · \\
\hline Geranium robertianum & . & . & . & . & . & . & + & . \\
\hline Eupatorium salviae & . & . & . & . & . & . & . & + \\
\hline Hydrocotyle volckmannii & . & . & . & . & . & . & . & + \\
\hline
\end{tabular}


BOSQUE 25(1): 69-85, 2004

Comparación fitosociológica de los bosques de Belloto (Beilschmiedia, Lauraceae) en Chile central

\section{CUADRO 2}

Estructura florística del bosque de Belloto del Sur.

Floristic structure of the Belloto del Sur forest.

\begin{tabular}{|c|c|c|c|c|c|c|c|c|c|c|}
\hline Censo $\mathrm{N}^{\mathrm{o}}$ & 1 & 2 & 3 & 4 & 5 & 6 & 7 & 8 & 9 & 10 \\
\hline Altitud (m s.n.m.) & 800 & 850 & 850 & 850 & 850 & 850 & 850 & 850 & 850 & 900 \\
\hline Exposición & SO & S & SE & SE & SO & SE & SO & SO & SE & SE \\
\hline Número de especies & 25 & 21 & 22 & 23 & 13 & 9 & 12 & 10 & 9 & 10 \\
\hline Beilschmiedia berteroana & 20 & 10 & 20 & 20 & 60 & 60 & 60 & 60 & 70 & 60 \\
\hline Lomatia dentata & 10 & + & 10 & + & + & 20 & 5 & + & 10 & . \\
\hline Cryptocarya alba & 10 & 5 & 10 & + & + & + & . & + & . & . \\
\hline Chusquea cumingii & + & + & + & + & + & . & + & . & . & . \\
\hline Citronella mucronata & . & 5 & + & + & + & + & 5 & . & . & . \\
\hline Nothofagus macrocarpa & . & . & . & 10 & + & 5 & . & + & 5 & + \\
\hline Hydrangea serratifolia & 5 & . & . & + & 5 & 10 & + & . & . & . \\
\hline Azara petiolaris & + & $\mathrm{r}$ & . & + & . & . & + & . & + & . \\
\hline Sophora macrocarpa & + & + & + & + & . & . & . & . & + & . \\
\hline Blechnum hastatum & + & . & . & + & . & + & + & + & . & . \\
\hline Aristotelia chilensis & . & + & + & + & . & . & . & . & + & + \\
\hline Luma apiculata & . & . & . & + & 15 & . & . & 20 & + & + \\
\hline Colliguaya salicifolia & + & + & + & + & . & . & . & . & . & . \\
\hline Adiantum chilense & + & + & + & . & . & . & + & . & . & . \\
\hline Muehlenbeckia hastulata & . & + & + & . & . & . & + & . & . & + \\
\hline Diplolepis menziesii & . & + & + & + & . & . & + & . & . & . \\
\hline Aextoxicon punctatum & . & . & + & + & + & 5 & . & . & . & . \\
\hline Chusquea culeou & . & . & . & + & . & . & . & + & + & + \\
\hline Ribes punctatum & . & + & + & + & . & . & . & . & . & . \\
\hline Adiantum chilensis var. & . & + & + & . & + & . & . & . & . & . \\
\hline Austrocedrus chilensis & . & . & 5 & + & + & . & . & . & . & . \\
\hline Lardizabala biternata & . & . & . & . & + & + & + & . & . & . \\
\hline Retanilla stricta & . & . & . & . & . & . & + & + & . & 5 \\
\hline Laurelia sempervirens & + & . & . & . & . & . & . & . & 5 & . \\
\hline Colliguaya dombeyana & + & . & + & . & . & . & . & . & . & . \\
\hline Plantago lanceolata & + & . & . & . & . & . & . & . & . & + \\
\hline Libertia sessiliflora & . & + & . & + & . & . & . & . & . & . \\
\hline Carex $s p$ & . & + & + & . & . & . & . & . & . & . \\
\hline Senecio sp. & . & . & + & + & . & . & . & . & . & . \\
\hline Megalastrum spectabile & . & . & . & + & + & . & . & . & . & . \\
\hline Tristerix tetrandrus & . & . & . & . & . & . & . & + & . & + \\
\hline Nothofagus obliqua & 5 & . & . & . & . & . & . & . & . & . \\
\hline Nothofagus glauca & 5 & . & . & . & . & . & . & . & . & . \\
\hline Prostia pyrifolia & + & . & . & . & . & . & . & . & . & . \\
\hline Lithrea caustica & + & . & . & . & . & . & . & . & & . \\
\hline Adesmia denticulata & + & . & . & . & . & . & . & . & . & . \\
\hline Viviania marifolia & + & . & . & . & . & . & . & . & . & . \\
\hline Cerastium arvense & + & . & . & . & . & . & . & . & . & . \\
\hline
\end{tabular}




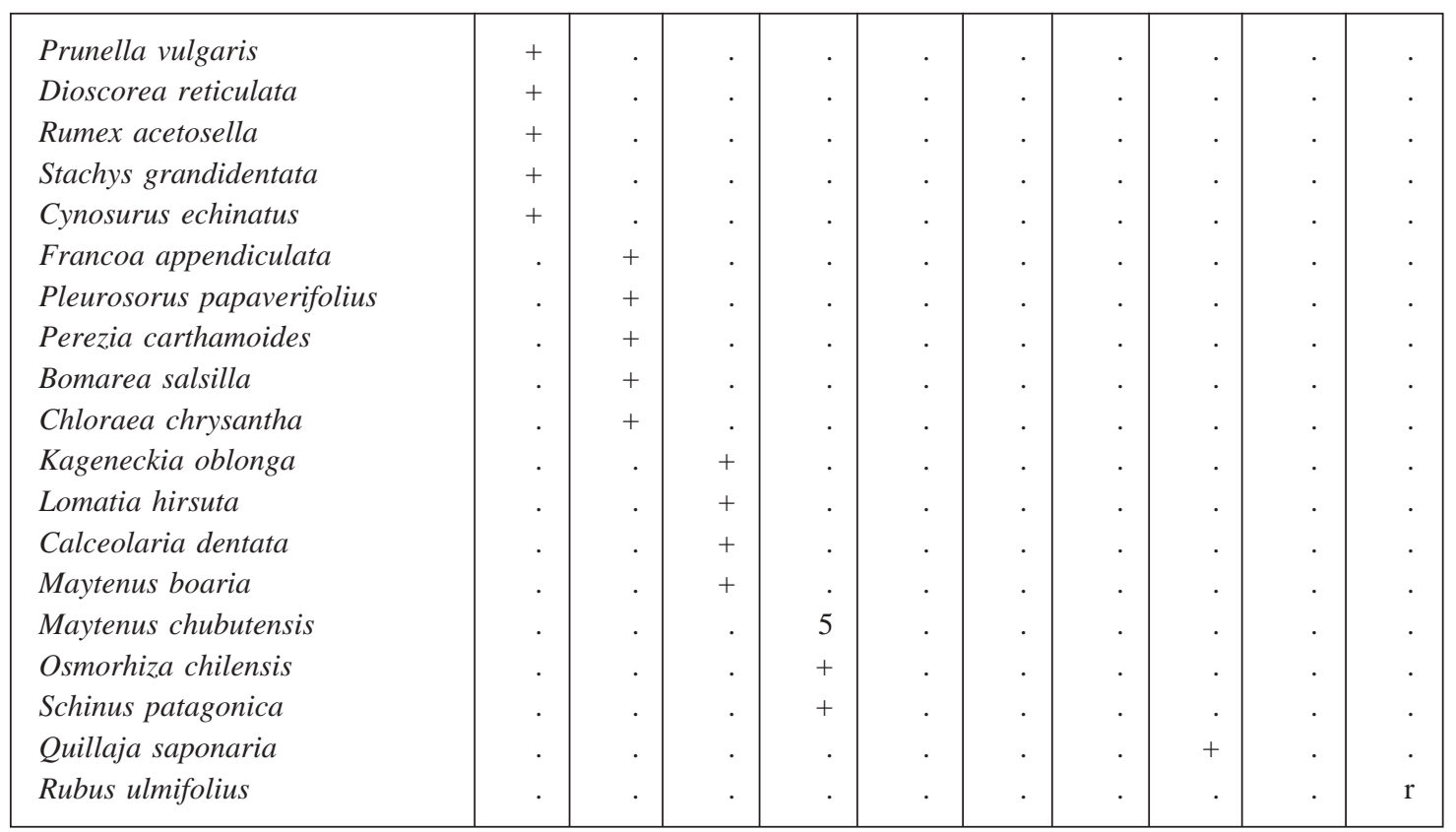

Flora. El promedio de especies por censo fue mayor en los bosques de Belloto del Norte (25, $25)$ que en los de Belloto del Sur $(15,40)$. No obstante lo anterior, el número de especies autóctonas es prácticamente igual en ambas asociaciones (51 y 52), de manera que el mayor número de especies del bosque de Belloto del Norte sólo se debe a la presencia de malezas alóctonas de escasa cobertura (figura 3 y cuadro 3 ).

La diferencia florística entre ambos bosques es grande, ya que de las 124 especies presentes en la tabla fitosociológica sólo $12(14,88 \%)$ son comunes, a saber: Lithrea caustica (Mol.) H. et A., Cryptocarya alba (Mol.) Looser, Lardizabala biternata R. et P., Proustia pyrifolia DC, Quillaja saponaria Mol., Citronella mucronata (R. et P.) D.Don, Maytenus boaria Mol., Diplolepis menziessii Schult., Stachys grandidentata Lindl., Aristotelia chilensis (Mol.) Stuntz., Calceolaria dentata R. et P. y Plantago lanceolata L. De ellas las 5 primeras corresponden a especies esclerófilas y la última es una maleza de origen europeo.

Las especies más importantes del bosque de Belloto del Norte, de acuerdo a su frecuencia y cobertura son el Belloto del Norte, que es seguido en importancia por Myrceugenia exsucca, un árbol propio de bosques pantanosos, y con menor importancia, Schinus latifolius (Gill. ex Lindl.) Engler, y Cryptocarya alba, propias de los bos- ques esclerófilos más húmedos de Chile central (cuadro 4). El quinto lugar lo ocupa Blepharacalyx cruckshanksii (H. et A.) Kausel, otra especie de bosques pantanosos. Del resto, Lithrea caustica y Peumus boldus Mol. son árboles esclerófilos; Cestrum parqui L'Herit, un arbusto, y Adiantum chilense Kaulf. var. hirsutum Hook et Grev., un helecho de lugares sombríos. Por último, Hydrocotyle chilense var. hirstutum L. f. es una hierba alóctona que coloniza agua corriente eutroficada. La importancia de esta especie también indica intervención antrópica de los rodales.

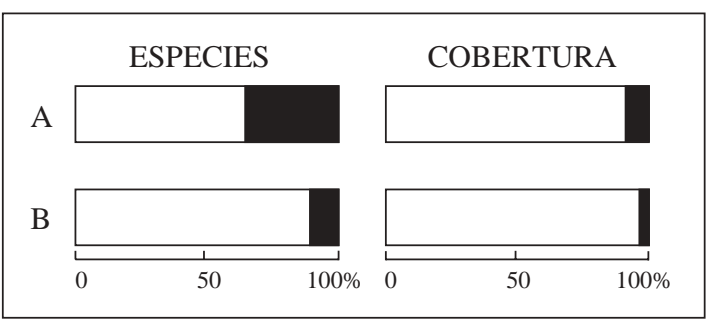

Figura 3. Origen fitogeográfico de la flora de los bosques de Belloto del Norte (A) y de Belloto del Sur (B), según número de especies y cobertura. Las barras blancas corresponden a especies autóctonas y las negras a alóctonas.

Phytogeographical origin of the flora of the Belloto del Norte (A) and Belloto del Sur (B) forests with respect to species number and cover. The white bars indicate autochthonous species and the black bars alochthonous species. 
BOSQUE 25(1): 69-85, 2004

Comparación fitosociológica de los bosques de Belloto (Beilschmiedia, Lauraceae) en Chile central

\section{CUADRO 3}

Número y cobertura de especies vegetales y su origen fitogeográfico. Number and cover of plant species and their phytogeographical origin.

\begin{tabular}{|l|c|c|c|r|}
\hline \multirow{2}{*}{ Bosque de Origen } & \multicolumn{2}{|c|}{ Belloto del Norte } & \multicolumn{2}{c|}{ Belloto del Sur } \\
\hline \multirow{3}{*}{ Autóctonas } & Especies & Cobertura & Especies & Cobertura \\
\cline { 2 - 5 } Alóctonas & 51 & 769 & 52 & 755 \\
\hline Total & 28 & 84 & 5 & 5 \\
\hline
\end{tabular}

\section{CUADRO 4}

Espectro biológico por especies y cobertura de los bosques estudiados. Biological spectra of the studied forests with respect to the number and species cover.

\begin{tabular}{|l|c|c|c|c|}
\hline \multirow{2}{*}{ Bosque de Origen } & \multicolumn{2}{|c|}{ Belloto del Norte } & \multicolumn{2}{c|}{ Belloto del Sur } \\
\hline Forma de vida & Especies & Cobertura & Especies & Cobertura \\
\hline Fanerófitos & 26 & 685 & 36 & 726 \\
Caméfitos & 8 & 16 & 5 & 5 \\
Hemicriptófitos & 15 & 56 & 3 & 3 \\
Criptófitos & 10 & 52 & 3 & 3 \\
Terófitos & 19 & 44 & 57 & 760 \\
\hline Total & 78 & 853 & & 30 \\
\hline
\end{tabular}

En el bosque de Belloto del Sur son importantes Beilschmiedia berteroana y Lomatia dentata (R. et P.) R. Br.; esta última es una Proteácea perennifolia de los bosques higrófilos templados chilenos (cuadro 4). El tercer lugar lo ocupa el árbol Cryptocarya alba y el cuarto nuevamente una especie mesófila del bosque valdiviano, Luma apiculata (DC) Burret. El quinto lugar de importancia corresponde al Roble del Norte: Nothofagus macrocarpa (DC) F.M. Vásquez \& R.A. Rodr., especie caducifolia, propia de los bosques caducifolios templados o bosques maulinos. Hydrangea serratifolia (H. et A.) F. Phil. es una trepadora de los bosques valdivianos y Citronella mucronata (R. et P.) D.Don, una especie esclerófila que puede ocupar ambientes con humedad edáfica. Los tres últimos son arbustos que proliferan al ralearse el dosel superior.

Espectro biológico. En el espectro biológico de las asociaciones comparadas dominan los fanerófitos leñosos y también son importantes los hemicriptófitos o hierbas perennes, lo que corresponde a un típico espectro de formaciones boscosas (cuadro 5). La abundancia de terófitos (hierbas anuales) en el bosque de Belloto del Norte indica un clima más seco, lo que también podría deberse a una mayor insolación por apertura del dosel. Esto señalaría intervención humana en los rodales estudiados. La presencia de criptófitos acuáticos se debe a la existencia de agua empozada bajo el dosel o en el borde de los rodales. 


\section{CUADRO 5}

Especies con mayor valor de importancia en los bosques de Belloto. Species with a greater importance value in both Belloto forests.

\begin{tabular}{|c|c|c|c|}
\hline Especie & Frecuencia & Cobertura & Valor de Importancia \\
\hline $\begin{array}{l}\text { Bosque de Belloto del Norte: } \\
\text { Beilschmiedia miersii } \\
\text { Myrceugenia exsucca } \\
\text { Schinus latifolia } \\
\text { Cryptocarya alba } \\
\text { Blepharocalyx cruckshanksii } \\
\text { Lithraea caustica } \\
\text { Adiantum chilense var. } \\
\text { Peumus boldus } \\
\text { Hydrocotyle ranunculoides } \\
\text { Cestrum parqui }\end{array}$ & $\begin{array}{l}8 \\
4 \\
7 \\
6 \\
6 \\
6 \\
5 \\
6 \\
3 \\
6\end{array}$ & $\begin{array}{r}300 \\
91 \\
68 \\
61 \\
38 \\
38 \\
24 \\
14 \\
26 \\
6\end{array}$ & $\begin{array}{r}39,08 \\
12,62 \\
11,40 \\
10,09 \\
7,39 \\
7,39 \\
5,26 \\
4,58 \\
4,51 \\
3,64\end{array}$ \\
\hline $\begin{array}{l}\text { Bosque de Belloto del Sur: } \\
\text { Beilschmiedia berteroana } \\
\text { Lomatia dentata } \\
\text { Cryptocarya alba } \\
\text { Luma apiculata } \\
\text { Nothofagus macrocarpa } \\
\text { Hydrangea serratifolia } \\
\text { Citronella mucronata } \\
\text { Chusquea cumingii } \\
\text { Azara petiolaris } \\
\text { Sophora macrocarpa }\end{array}$ & $\begin{array}{r}10 \\
9 \\
7 \\
5 \\
6 \\
5 \\
6 \\
6 \\
5 \\
5\end{array}$ & $\begin{array}{r}440 \\
59 \\
29 \\
38 \\
23 \\
22 \\
14 \\
6 \\
5 \\
5\end{array}$ & $\begin{array}{r}64,34 \\
13,57 \\
8,34 \\
8,23 \\
6,90 \\
6,12 \\
5,71 \\
4,66 \\
3,89 \\
3,89\end{array}$ \\
\hline
\end{tabular}

Al considerar la cobertura de cada forma de vida, el espectro biológico del bosque del Belloto del Sur se simplifica mucho, lo que es propio de condiciones de bosque higrófilo. Por el contrario, en el bosque de Belloto del Norte, las demás formas de vida también tienen importancia al considerar la cobertura de sus individuos (figura 4), lo que más bien corresponde a una comunidad arbustiva de matorral.

Análisis estadístico multivariado. Los censos de ambas comunidades boscosas se unen con una similitud florística menor al 50\% (figura 5). También se observa una mayor afinidad florística entre los censos en el bosque de Belloto del Sur, con un valor superior al $80 \%$. Los censos de vegetación del bosque de Belloto del Norte presentaron una similitud florística cercana al $70 \%$. Esta mayor heterogeneidad florística de los censos del bosque de Belloto del Norte puede tener su origen en la mayor intervención antrópica de sus rodales

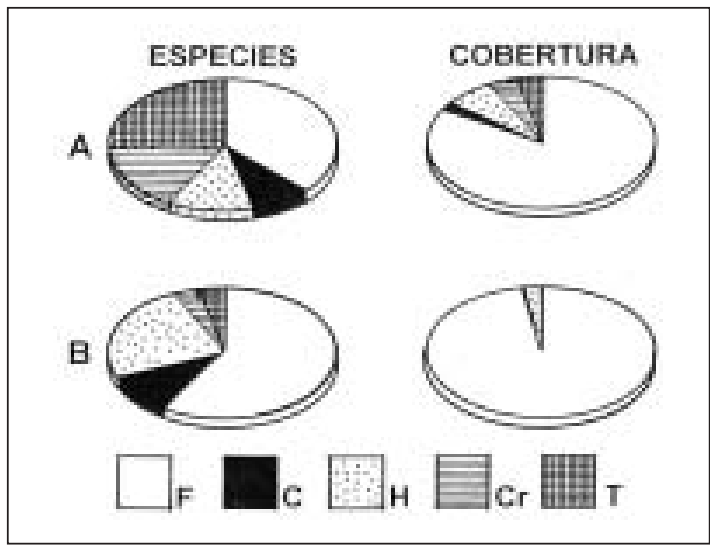

Figura 4. Espectro biológico de los bosques de Belloto del Norte (A) y de Belloto del Sur (B) expresado en número de especies y cobertura. Formas de vida: $\mathrm{F}=$ fanerófitos, $\mathrm{C}=$ caméfitos, $\mathrm{H}=$ hemicriptófitos, $\mathrm{Cr}=$ criptófitos y $\mathrm{T}=$ terófitos.

Biological spectra of the flora of the Belloto del Norte (A) and Belloto del Sur (B) forests with respect to species number and cover. Life forms: $\mathrm{F}=$ phanerophytes, $\mathrm{C}=$ chamaphytes, $\mathrm{H}=$ hemicryptophytes, $\mathrm{Cr}=$ cryptophytes and $\mathrm{T}=$ therophytes. 
BOSQUE 25(1): 69-85, 2004

Comparación fitosociológica de los bosques de Belloto (Beilschmiedia, Lauraceae) en Chile central

debido a su cercanía a la costa y su mayor accesibilidad.

La mayoría de las especies se ordenan en la intersección de los dos primeros componentes principales que explican el 71,61\% de la variación (figura 6). Sólo ocho especies se segregan de este grupo central. Entre ellas, Beilschmiedia berteroana es segregada hacia el extremo derecho del primer componente principal, separándose claramente del resto. De acuerdo a los requerimientos de sitios de las especies segregadas, este eje podría corresponder a una gradiente de temperatura ambiental que aumentaría hacia la izquierda de la figura. Esta interpretación está de acuerdo con la mayor altitud en que crece $B$. berteroana, en comparación con B. miersii (2).

El segundo componente principal, que sólo explica el $28,34 \%$ de la varianza, separa el resto de estas especies en un gradiente cuyos extremos son ocupados por B. miersii y Citronella mucronata. De acuerdo a los requerimientos de sitio de estas dos especies, este gradiente podría asimilarse a una variación de la humedad ambiental, la que aumentaría hacia arriba, mientras que hacia abajo disminuiría.

$\mathrm{Al}$ ordenar los censos, en los dos primeros componentes principales se observa una nítida separación entre los de ambas comunidades boscosas, ubicándose los del bosque de Belloto del Sur en el extremo derecho del primer componente principal

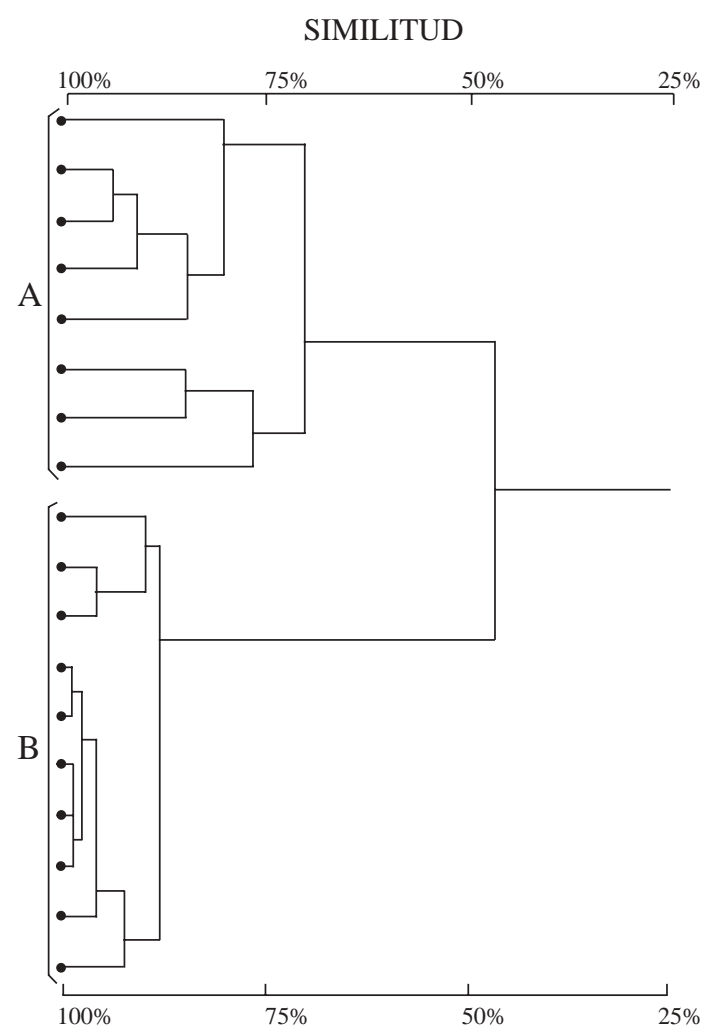

Figura 5. Dendrograma de similitud florística entre los censos de los bosques de Belloto del Norte (A) y de Belloto del Sur (B).

Dendrogram of floristic similarities of vegetation samples from the Belloto del Norte (A) and Belloto del Sur (B) forests.

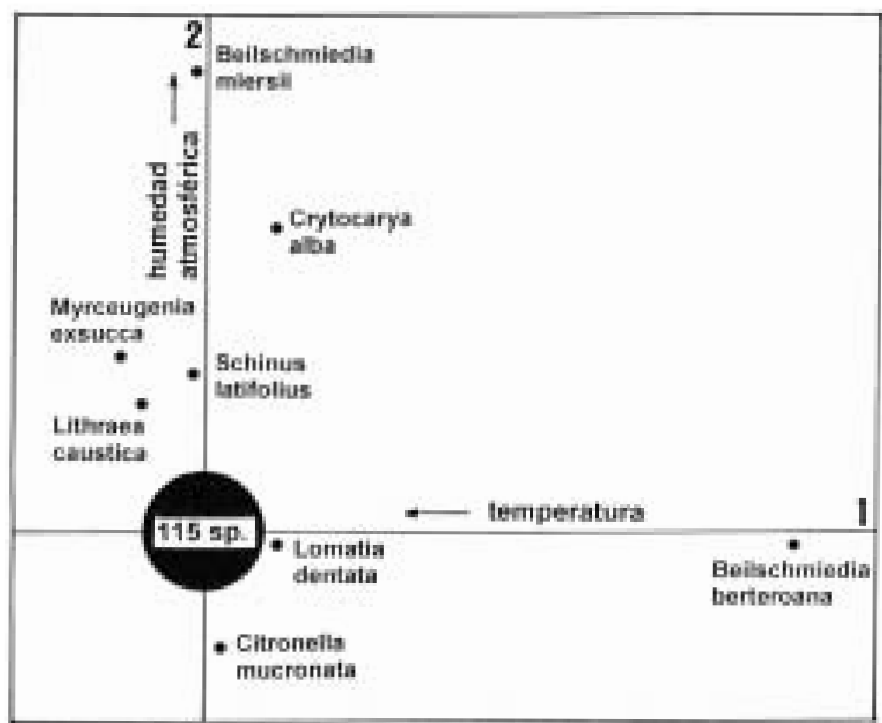

Figura 6. Distribución de las especies de los bosques de Belloto en el plano formado por los dos primeros componentes principales. Distribution of species in the Belloto forests in the plane formed by the first two principal components. 
(eje horizontal) y los del Belloto del Norte en el extremo superior del segundo eje vertical (figura 7). En esta ordenación también queda de manifiesto la mayor homogeneidad florística de los censos de los bosques de Belloto del Sur, que se ordenan más próximos entre sí.

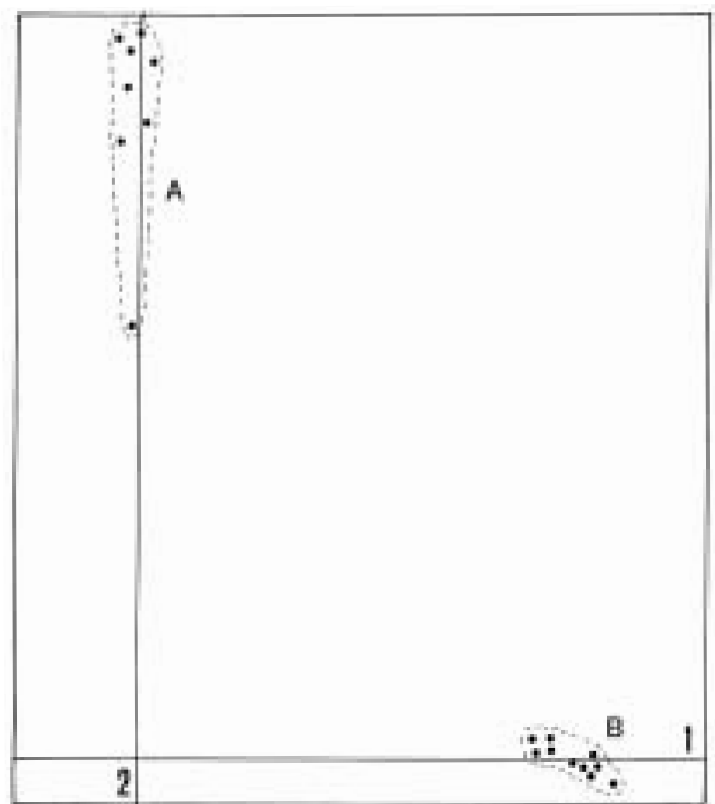

Figura 7. Distribución de los censos de vegetación de los bosques de Belloto del Norte (A) y de Belloto del Sur (B) en el plano formado por los dos primeros componentes principales.

Distribution of vegetation samples in the Belloto del Norte (A) and Belloto del Sur (B) forests in the plane formed by the first two principal components.

\section{CONCLUSIONES Y DISCUSION}

La gran diferencia macroclimática en las áreas de distribución de ambos bosques explicaría la baja similitud florística entre ellos. Sin embargo, algunas especies esclerófilas de lugares húmedos son compartidas, debido seguramente a la similitud en las condiciones de estos hábitats protegidos en el fondo de quebradas $(29,30)$.

El espectro biológico de ambas comunidades boscosas está dominado por especies leñosas y hierbas perennes, lo que es propio de bosques; sin embargo, en los bosques de Belloto del Norte aparece también una gran cantidad de hierbas anuales con escasa cobertura, lo que indica un aumento del xerofitismo en ellos (31), seguramente causado por la apertura antropogénica del dosel.
En los bosques del Belloto del Sur se encontró una mayor homogeneidad florística que en aquellos del Belloto del Norte, seguramente debido a la mayor presencia de malezas anuales alóctonas en este último. Esa mayor homogeneidad habla de una mejor conservación de los rodales estudiados.

Los bosques de Belloto del Norte habían sido descritos como una asociación boscosa por Schmithüsen (32), con el nombre de Beilschmiedietum miersii. Los bosques de Belloto del Sur no han recibido una denominación fitosociológica válida. Litton et al. (6) estudiaron el mismo lugar trabajado en este estudio, pero aunque hacen una clasificación estadística de las parcelas, no presentan una ordenación fitosociológica de ellas. No obstante, entregan una clasificación en dos asociaciones y cuatro subasociaciones que reciben el nombre de variantes. Estimamos que no es posible realizar una clasificación fitosociológica del análisis de un rodal $\mathrm{y}$, que al parecer, las unidades descritas son más bien el resultado de haber incluido zonas ecotonales o estados de degradación en el muestreo. De la misma manera dichos autores utilizan la especie Nothofagus obliqua var. obliqua (Roble) para la nomenclatura fitosociológica que proponen, pero la especie más frecuente y abundante en los bosques de Belloto del Sur es Nothofagus macrocarpa (Roble del Norte o de Santiago), nombre nuevo para Nothofagus obliqua (Mirb.) Oerst. var. macrocarpa (A. DC.) Reiche, propuesto por Vázquez y Rodríguez (33).

Por lo anterior, proponemos la denominación de Nothofago-Beilschmiedietum berteroanae ass. nova para los bosques de Belloto del Sur y designamos como censo tipo el $\mathrm{N}^{\mathrm{o}} 6$ de la tabla fitosociológica (cuadro 2). En el cuadro 6 se entrega la clasificación sintaxonómica de las dos asociaciones boscosas estudiadas: el Beilschmiedietum miersii integrado a la clasificación de los bosques esclerófilos de Chile central y el NothofagoBeilschmiedietum berteroanae a los bosques higrófilos templados de la Clase Wintero-Nothofagetea (34) en la nueva Subalianza Nothofagenion glauco-alessandri establecida por Amigo et al. (13).

En los bosques de Belloto del Norte influye mucho el agua edáfica, como lo confirma la presencia de especies leñosas de Mirtáceas propias de los bosques pantanosos (Hualves) de Chile central (35) que presentan un carácter azonal $(36,37)$, tales como Blepharocalyx cruckshanksii, Myrceugenia exsucca (DC) Berg. y Luma chequen 
BOSQUE 25(1): 69-85, 2004

Comparación fitosociológica de los bosques de Belloto (Beilschmiedia, Lauraceae) en Chile central

\section{CUADRO 6}

Clasificación sintaxonómica de los Bosques de Belloto chilenos.

Sintaxonomical classification of the Chilean Belloto forests.

\begin{tabular}{|c|c|}
\hline Sintaxa & Bosque de Belloto del Norte \\
\hline Clase & $\begin{array}{l}\text { Lythraeo-Cryptocaryetea albae Oberd, } \\
1960 .\end{array}$ \\
\hline Orden & Cryptocaryetalia albae Schmith, 1954. \\
\hline Alianza & Cryptocaryon albae Schmith, 1954. \\
\hline Subalianza & \\
\hline Asociación & Beilschmiedietum miersii Schmith, 1954. \\
\hline Sintaxa & Bosque de Belloto del Sur \\
\hline Clase & Wintero-Nothofagetea Oberd, 1960. \\
\hline Orden & Laurielietalia philippianae Oberd, 1960. \\
\hline Alianza & $\begin{array}{l}\text { Nothofago-Eucryphion cordifoliae } \\
\text { Oberd, } 1960 .\end{array}$ \\
\hline Subalianza & $\begin{array}{l}\text { Nothofagenion glauco-alessandri Amigo, } \\
\text { San Martín \& Quint, } 2000 .\end{array}$ \\
\hline Asociación & $\begin{array}{l}\text { Nothofago-Beilschmiedietum berteroanae } \\
\text { ass. nova. }\end{array}$ \\
\hline
\end{tabular}

(Mo.) A. Gray $(38,39)$. En los bosques de Belloto del Sur el carácter azonal, propio de quebradas, lo entregan las especies de los bosques higrófilos templados, cuya área de distribución es mucho más austral, entre ellas, Laurelia sempervirens (R. et P.) Tul., Hydrangea serratifolia, Luma apiculata, Aextoxicon punctatum (R. et P.) y Lomatia dentata. Aunque esta última tiene una amplia distribución latitudinal, es más bien un hemixerófito pionero en los bosques valdivianos, por la presencia de conglomerados proteiformes en su sistema radical (40).

Dentro de su área de distribución el Belloto del Norte se presenta en rodales boscosos del Beilschmiedietum miersii, muy intervenidos $(5,41)$. Por el contrario, el Belloto del Sur sólo aparece formando bosques en el lugar estudiado, ya que en los otros lugares citados por la literatura dentro de su área se presenta como árboles aislados remanentes del bosque original (7, 42, 43). Según San Martín et al. (44) los patrones de crecimiento vegetativo, de floración y fructificación de esta especie están adaptados a condiciones de clima templado. Efectivamente los Bosques de Belloto del Sur crecen a mayor altitud que los de Belloto del Norte. No obstante lo anterior, ante una intervención antrópica permanente ellos podrían desaparecer, por el dominio de especies caducifolias de rápido crecimiento, en este caso por Nothofagus macrocarpa, que haría desaparecer las especies laurifolias ante un aumento del xerofitismo (45).

Dentro del área de distribución de Belloto del Norte los fragmentos de bosque que conforma se encuentran rodeados por bosques esclerófilos o sus estados de degradación, como el "Espinal" de Acacia caven Mol. Los bosques de Belloto del Sur, por el contrario, se encuentran inmersos en una zona donde predominan los bosques caducifolios de Nothofagus, aunque originalmente también ocupó suelos de uso agrícola potencialmente de bosques esclerófilos (46).

Actualmente, rodales de ambos bosques están incluidos en el Sistema Nacional de Areas Silvestres Protegidas por el Estado de Chile (SNASPE) que supervisa la Corporación Nacional Forestal (CONAF) (43, 47-49).

\section{AGRADECIMIENTOS}

Se agradece la valiosa ayuda de la CONAF que en ambos lugares colocó a nuestra disposición apoyo de personal y transporte. También agradecemos a la Dirección de Investigación y Desarrollo de la Universidad Austral de Chile (Proyecto DID-UACH No S-97-05) y al Servicio Alemán de Intercambio Académico (DAAD) que facilitó la revisión de la literatura europea.

\section{BIBLIOGRAFIA}

(1) KOSTERMANS, A. Las lauráceas chilenas. Revista Universitaria (Chile), 1939, Vol. 24, No 1, p. 201-232.

(2) RODRIGUEZ, R., M. QUEZADA, O. MATTHEI. Flora arbórea de Chile. Concepción: Editorial Universidad de Concepción. 1983. 360 p.

(3) RUNDEL, P. The matorral zone of central Chile. In: DI CASTRI, F., GOODALL, D. W., R. L. SPECHT. Mediterranean Type Shrublands. Amsterdam: Elsevier Scientific Pub. 1981, p. 175-201.

(4) QUINTANILLA, V. Carta fitogeográfica de Chile mediterráneo. Contribuciones Científicas y Tecnológicas Area Geociencias USACH (Chile), 1987, Vol. 70, p. 1-29.

(5) BALDUZZI, A., R. TOMASELli, I. SEREY, R. VILLASEÑOR. New Phytosociological observations on the Mediterranean type of climax vegetation of central Chile. Atti. Ist. Bot. Lab. Critt. Univ. Pavia (Italia), 1981, Vol. 6, No 14 , p. 93-112. 
(6) LITTON, C., J. DIAZ, R. LARA. Composición florística y estructura vertical de un rodal de Beilschmiedia berteroana (Gay) Kosterm. en la precordillera andina de la VII Región de Chile. Bosque (Chile), 1997, Vol. 18, $\mathrm{N}^{\mathrm{o}} 1$, p. 61-72.

(7) VILLA, I. Prospección de las especies arbóreas en peligro de extinción y las acciones llevadas a cabo por la Corporación Nacional Forestal, para su protección en la Región del Maule. II Encuentro Científico sobre el Medio Ambiente. Talca (Chile) Versiones Abreviadas, 1986, Vol. 1, p. 365-370.

(8) BENOIT, I. Red book on Chilean terrestrial flora. Ministerio de Agricultura, Corporación Nacional Forestal (CONAF, Chile). 1989, $151 \mathrm{p}$

(9) SAN MARTIN, J., C. RAMIREZ. Fitosociología de los Nothofagus de la zona mesomórfica chilena. Bosque (Chile), 1987, Vol. 8, No 2, p. 121-125.

(10) GAJARDO, R. La vegetación natural de Chile. Santiago: Editorial Universitaria. 1994. 165 p.

(11) SCHMITHÜSEN, J. Die räumliche Ordnung der chilenischen Vegetation. Bonner Geographische Abhandlungen, 1956, Vol. 17, p. 1-89.

(12) OBERDORFER, E. Pflanzensoziologische Studien in Chile - Ein Vergleich mit Europe. Flora et Vegetatio Mundi, 1960, Vol. 2, p. 1-208.

(13) AMIGO, J., J. SAN MARTIN, L. G. QUINTANILLA. Estudio fitosociológico de los bosques de Nothofagus glauca (Phil.) Krasser del Centro-Sur de Chile. Phytocoenologia, 2000, Vol. 30, $\mathrm{N}^{\circ} 2$, p. 193-221.

(14) DI CASTRI, F., E. HAJEK. Bioclimatología de Chile. Santiago: Universidad Católica de Chile. 1976. 128 p.

(15) AMIGO, J., C. RAMIREZ. A bioclimatic classification of Chile: woodland communities in the temperate zone. Plant Ecology, 1998, Vol. 136, p. 9-26.

(16) HAJEK, E., F. DI CASTRI. Bioclimatografía de Chile. Santiago: Universidad Católica de Chile. 1975. 214 p.

(17) DIERSCHCKE, H. Pflanzensoziologie - Grundlagen und Methoden. Stuttgart: Eugen Ulmer. 1994. 683 p.

(18) RAMIREZ, C., C. SAN MARTIN, P. OJEDA. Muestreo y tabulación fitosociológica aplicados al estudio de los bosques nativos. Bosque (Chile), 1997, Vol. 18, № 2, p. 19-27.

(19) KNAPP, R. Considerations on quantitative parameters and qualitative attributes in vegetation analysis and in phytosociological relevés. Vol. 8, La Haya: Dr. W. Junk Publishers, 1984. Sampling methods and taxon analysis in vegetation science, p. 77-119.

(20) MARTICORENA, C., M. QUEZADA. Catálogo de la flora vascular de Chile. Gayana Botanica (Chile), 1985, Vol. 42, No $1 / 2$, p. 5-157.

(21) MUELLER-DOMBOIS, D., H. ELLENBERG. Aims and methods of vegetation ecology. New York: John Wiley \& Sons. 1974. 547 p.

(22) WIKUM, D., G. F. SHANHOLTZER. Application of the Braun-Blanquet cover-abundance scale for vegetation analysis in land development studies. Environmental Management, 1978, Vol. 2, No 4, p. 323-329.

(23) SAN MARTIN, C., C. RAMIREZ, H. FIGUEROA. Análisis multivariable de la vegetación de un complejo de turberas en Cordillera Pelada (Valdivia, Chile). Lazaroa (España), 1999, Vol. 20, p. 95-106.

(24) LUDWIG, J., J. REYNOLDS. Statistical ecology - A primer on methods and computing. New York: John Wiley \& Sons. 1988. 337 p.

(25) KENT, M., P. COOKER. Vegetation description and analysis. Chichester: John Wiley \& Sons. 1995. 363 p.
(26) SAIZ, F. Experiencias en el uso de criterios de similitud en el estudio de comunidades. Arch. Biol. Med. Exp. (Chile), 1980, Vol. 13, p. 387-402.

(27) DIGBY, P. G. N., R. A. KEMPTON. Multivariate analysis of ecological communities. London: Chapman and Hall. 1987. 205 p.

(28) BARKMANN, J., J. MORAVEC, S., RAUSCHERT. Code of phytosociological nomenclature. Vegetatio, 1986 Vol. 67, No 3, p. 145-195.

(29) BALDUZZI, A., R. TOMASELLI, I. SEREY, R. VILLASEÑOR. Degradation of the mediterranean type of vegetation in central Chile. Ecología mediterránea, 1982, Vol. 8, $\mathrm{N}^{\circ} 1 / 2$, p. 223-240.

(30) DONOSO, C. Reseña ecológica de los bosques mediterráneos de Chile. Bosque (Chile), Vol. 4, No 2, 1982 p. 117-146.

(31) RAMIREZ, C., C. SAN MARTIN, P. OJEDA. Estudio de la flora de comunidades ruderales antropogénicas en la IX Región de la Araucanía, Chile. Stvdia Botánica (Salamanca), 1999, Vol. 18, p. 47-68.

(32) SCHMITHÜSEN, J. Waldgesellschaften des nördlichen Mittelchile. Vegetatio, 1954, Vol. 5/6, p. 479-486.

(33) VAZQUEZ, F., R. RODRIGUEZ. A new subspecies and two new combinations of Nothofagus Blume (Nothofagaceae) from Chile. Botanical Journal of the Linnean Society, 1999, Vol. 129, p. 75-83.

(34) TOMASELLI, R. The longitudinal zoning of vegetation in the southern sector of the Andes. Studi Trentini di Scienze Naturali Acta Biologica, 1981, Vol. 58, p. 471-484.

(35) SAN MARTIN, J., A. TRONCOSO, C. RAMIREZ, C. SAN MARTIN, A. DUARTE. Estudio florístico y vegetacional de los bosques pantanosos nativos de la cordillera costera entre los ríos Rapel y Mataquito. Revista Geográfica de Chile Terra Australis, 1990, Vol. 33, p. 103-128.

(36) WALTER, H. Vegetationszonen und Klima. Stuttgart: Eugen Ulmer. 1970. 244 p.

(37) HUECK, K. Los bosques de Sudamérica - Ecología, composición e importancia. Eschborn: GTZ-Verlag. 1978. $476 \mathrm{p}$.

(38) SAN MARTIN. J., J. SOLERVICENS, C. RAMIREZ, C. SAN MARTIN, M. ELGUETA. Estudio fitosociológico de los bosques pantanosos de Mirtáceas de la Región del Maule, Chile. Ciencias Forestales (Chile), 1992, Vol. 8, $\mathrm{N}^{\mathrm{o}} 1 / 2$, p. 3-18.

(39) RAMIREZ, C., C. SAN MARTIN, J. SAN MARTIN Estructura florística de los bosques pantanosos de Chile central. En: J. ARMESTO, M. T. KALIN-ARROYO, C. VILLAGRAN. Ecología del bosque nativo en Chile. Santiago: Editorial Universitaria, 1996, p. 215-234.

(40) RAMIREZ, C., J. GRINBERGS, E. VALENZUELA, C. SAN MARTIN. Influencia de las raíces proteiformes en el desarrollo de plántulas de Gevuina avellana Mol. (Proteaceae). Bosque (Chile), 1990, Vol. 11, No 1, p. 11-20.

(41) QUINTANILLA, V. Fitogeografía de la cuenca de Quillota. Revista Geográfica de Valparaíso (Chile), 1976 , Vol. 7, p. 33-79.

(42) BENOIT, I. El Belloto del Sur. Chile Forestal (Chile), 1986, Vol. 128, p. 16-18.

(43) MUÑNOZ, M., H. NUÑEZ, J. YAÑEZ. Libro rojo de los sitios prioritarios para la conservación de la diversidad biológica en Chile. Ministerio de Agricultura, Corporación Nacional Forestal (CONAF) (Chile). 1996. 203 p.

(44) SAN MARTIN, J., A. VILLA, C. RAMIREZ. Fenología y crecimiento vegetativo de Beilschmiedia berteroana (Gay) Kosterm. en la precordillera andina de Chile central (35' 52' S / 71 06' W). Bosque (Chile), 2002, Vol. 23 , $\mathrm{N}^{\mathrm{o}} 1$, p. $37-45$. 
BOSQUE 25(1): 69-85, 2004

Comparación fitosociológica de los bosques de Belloto (Beilschmiedia, Lauraceae) en Chile central

(45) FRANK, D., M. FINCKH. Laurophyllisation of deciduous Nothofagus-Forest in Southern Chile. In: F. KLÖTLI, F. WALTHER, G. R. Recent shifts in vegetation boundaries of deciduous forest. Basel: Birkhäuser Verlag. 1968, p. 317-331.

(46) ESPINOZA, M. Apuntes botánicos sobre el belloto chileno de frutos comestibles. Boletín del Museo Nacional de Historia Natural, 1941, Vol. 19, p. 9-18.

(47) RUNDEL, P., P. WEISSER. La Campana, a new national park in central Chile. Biological Conservation, 1975, Vol. 8, p. 35-46.
(48) VILLASEÑOR, R., I. SEREY. Estudio fitosociológico de la vegetación del cerro La Campana (Parque Nacional La Campana) en Chile central. Atti. Ist. Bot. Lab. Critt. Univ. Pavia, 1981, Vol. 6, No 14, p. 69-91.

(49) ARROYO, M. T. K., O. MATTHEI, C. MARTICORENA, M. MUÑOZ, F. PEREZ, A. M. HUMAÑA. The vascular plant flora of the Bellotos del Melado national reserve, VII Región, Chile: A documented checklist. Gayana Botanica (Chile), 2000, Vol. 57, N² 2, p. 117-139. 
Catálogo florístico de los bosques de Belloto chilenos. Se entrega CLASE, Especie y Autoridad, Familia, Nombre común, Forma de vida $(F)$ y Origen fitogeográfico $(\mathrm{O})$.

Floristic checklist of the Chilean Belloto forests: class, species, author, family, common name, life-forms (F) and phytogeographical origin $(\mathrm{O})$.

\begin{tabular}{|c|c|c|c|c|}
\hline Especie & Familia & Nombre común & $\mathrm{F}$ & $\mathrm{O}$ \\
\hline \multicolumn{5}{|l|}{ SPHENOPSIDA (Equisetos) } \\
\hline Equisetum bogotense $\mathrm{HBK}$. & Equisetaceae & Limpia plata & $\mathrm{Crg}$ & A \\
\hline FILICOPSIDA (Helechos) & & & & \\
\hline Adiantum chilense Kaulf. & Adiantaceae & Patita negra & $\mathrm{H}$ & A \\
\hline Adiantum sulphureum Kaulf. & Adiantaceae & Doradilla & $\mathrm{H}$ & A \\
\hline Adiantum chilense Kaulf. var. hirsutum Hook. et Grev. & Adiantaceae & Doradilla & $\mathrm{H}$ & A \\
\hline Azolla filiculoides Lam. & Azollaceae & Flor del pato & $\mathrm{Crf}$ & A \\
\hline Blechnum hastatum Kaulf. & Blechnaceae & Palmilla & $\mathrm{H}$ & A \\
\hline Megalastrum spectabile (Kaulf.) A.R.Sm. et RC Moran & Dryopteridaceae & Helecho & $\mathrm{H}$ & A \\
\hline Pleurosorus papaverifolius (Kunze) Fée & Aspleniaceae & No conocido & $\mathrm{H}$ & A \\
\hline PINOPSIDA (Coníferas) & & & & \\
\hline $\begin{array}{l}\text { Austrocedrus chilensis (D. Don) Pic. Ser. et Bizz. } \\
\text { MAGNOLIOPSIDA (Dicotiledóneas) }\end{array}$ & Cupressaceae & Ciprés de la Cordillera & $\mathrm{Fa}$ & A \\
\hline Acacia caven (Mol.) Mol. & Mimosaceae & Espino maulino & Far & A \\
\hline Adenopeltis serrata (W. Aiton) Johnst. & Euphorbiaceae & Lechón & Far & A \\
\hline Adesmia denticulata Clos. & Fabaceae & Retama & Far & A \\
\hline Aextoxicon punctatum R. et P. & Aextoxicaceae & Olivillo, Tique & $\mathrm{Fa}$ & A \\
\hline Argythamnia tricuspidata (Lam.) Muell.-Arg. & Euphorbiaceae & Ventosilla & Far & A \\
\hline Aristotelia chilensis (Mol.) Stuntz. & Elaeocarpaceae & Maqui & Far & A \\
\hline Azara celastrina D. Don & Flacourtiaceae & Lilén & Far & A \\
\hline Azara petiolaris (D. Don) Johnst. & Flacourtiaceae & Lilén & Far & A \\
\hline Baccharis linearis (R. et P.) Pers. & Asteraceae & Chilca & Far & A \\
\hline Beilschmiedia berteroana (Gay) Kosterm. & Lauraceae & Belloto del Sur & $\mathrm{Fa}$ & A \\
\hline Beilschmiedia mersii (Gay) Kosterm. & Lauraceae & Belloto del Norte & $\mathrm{Fa}$ & A \\
\hline Blepharocalyx cruckshanksii (H. et A.) Kausel & Myrtaceae & Temu & $\mathrm{Fa}$ & A \\
\hline Calceolaria dentata $\mathrm{R}$. et $\mathrm{P}$. & Scrophulariaceae & Topa-Topa & $\mathrm{C}$ & A \\
\hline Capsella bursa-pastoris (L.) Medik. & Brassicaceae & Mastuerzo & $\mathrm{H}$ & I \\
\hline Cerastium arvense $\mathrm{L}$. & Caryophyllaceae & Cuernecita & $\mathrm{T}$ & I \\
\hline Cestrum parqui L'Herit & Solanaceae & Palqui & Far & A \\
\hline Cissus striata $\mathrm{R}$. et P. & Vitaceae & Voqui naranjillo & $\mathrm{Ft}$ & A \\
\hline Citronella mucronata (R. et P.) D. Don & Icacinaceae & Huilli patagua & $\mathrm{Fa}$ & A \\
\hline Colliguaja dombeyana A.H.L. Juss. & Euphorbiaceae & Colliguay & Far & A \\
\hline Colliguaja salicifolia Gill. Et Hook. & Euphorbiaceae & Colliguay & Far & A \\
\hline Conyza bonariensis (L.) Cronq. & Asteraceae & Coniza & $\mathrm{T}$ & I \\
\hline Cryptocarya alba (Mol.) Looser & Lauraceae & Peumo & $\mathrm{Fa}$ & A \\
\hline Diplolepis menziesii Schult. & Asclepiadaceae & Voquicillo & $\mathrm{T}$ & A \\
\hline Eupatorium glechonophyllum Less. & Asteraceae & Salvia negra & $\mathrm{C}$ & A \\
\hline Eupatorium salvia Colla & Asteraceae & Salvia & $\mathrm{C}$ & A \\
\hline Euphorbia peplus L. & Euphorbiaceae & Pichoga & $\mathrm{T}$ & I \\
\hline Euphorbia portulacoides $L$. & Euphorbiaceae & Pichoa & $\mathrm{C}$ & A \\
\hline
\end{tabular}


BOSQUE 25(1): 69-85, 2004

Comparación fitosociológica de los bosques de Belloto (Beilschmiedia, Lauraceae) en Chile central

\begin{tabular}{|c|c|c|c|c|}
\hline Especie & Familia & Nombre común & $\mathrm{F}$ & $\mathrm{O}$ \\
\hline Francoa appendiculata Cav. & Saxifragaceae & Llaupangue & $\mathrm{H}$ & A \\
\hline Fuchsia lycioides Andr. & Onagraceae & Palo falso & Far & A \\
\hline Fumaria capreolata $\mathrm{L}$. & Fumariaceae & Hierba de la culebra & $\mathrm{T}$ & I \\
\hline Galium aparine $\mathrm{L}$. & Rubiaceae & Lengua de gato & $\mathrm{C}$ & I \\
\hline Gamochaeta americana (Mill.) Wedd. & Asteraceae & Vira-Vira & $\mathrm{H}$ & A \\
\hline Geranium core-core Steud. & Geraniaceae & Core Core & $\mathrm{T}$ & A \\
\hline Geranium robertianum $\mathrm{L}$. & Geraniaceae & Core Core & $\mathrm{T}$ & I \\
\hline Gratiola peruviana $\mathrm{L}$. & Scrophulariaceae & Contrayerba & $\mathrm{C}$ & A \\
\hline Hydrangea serratifolia (H. et A.) F. Phil. & Hydrangeaceae & Pehueldén & $\mathrm{Ft}$ & A \\
\hline Hydrocotyle ranunculoides L.f. & Apiaceae & Hierba de la plata & $\mathrm{Crn}$ & I \\
\hline Hydrocotyle volckmannii Phil. & Apiaceae & Sombrerito de agua & Cre & A \\
\hline Kageneckia oblonga $\mathrm{R}$. et $\mathrm{P}$. & Rosaceae & Bollén & $\mathrm{Fa}$ & A \\
\hline Lardizabala biternata $\mathrm{R}$. et P. & Lardizabalaceae & Cogüilera & $\mathrm{Ft}$ & A \\
\hline Laurelia sempervirens (R. et P.) Tul. & Monimiaceae & Laurel & $\mathrm{Fa}$ & A \\
\hline Lithrea caustica (Mol.) H. et A. & Anacardiaceae & Litre & $\mathrm{Fa}$ & A \\
\hline Lomatia dentata (R. et P.) R. br. & Proteaceae & Piñol & $\mathrm{Fa}$ & A \\
\hline Lomatia hirsuta (Lam.) Diels ex Macbr. & Proteaceae & Radal & $\mathrm{Fa}$ & A \\
\hline Luma apiculata (DC) Burret & Myrtaceae & Arrayán & $\mathrm{Fa}$ & A \\
\hline Luma chequen (Mol.) A. Gray & Myrtaceae & Chequén & $\mathrm{Fa}$ & A \\
\hline Lythrum album H.B.K. & Lythraceae & Romerillo & $\mathrm{T}$ & I \\
\hline Maytenus boaria Mol. & Celastraceae & Maitén & $\mathrm{Fa}$ & A \\
\hline Maytenus chubutensis (Speg.) Lourt., O. Don et Sleum. & Celastraceae & Maitén del Chubut & Far & A \\
\hline Mimulus glabratus H.B.K. & Scrophulariaceae & Berro & $\mathrm{H}$ & A \\
\hline Muehlenbeckia hastulata (J.E.Sm.) Johnst. & Polygonaceae & Voqui quilo & $\mathrm{Ft}$ & A \\
\hline Myrceugenia exsucca (DC) Berg. & Myrtaceae & Petra & $\mathrm{Fa}$ & A \\
\hline Myrceugenia obtusa (DC) Berg. & Myrtaceae & Arrayán & Far & A \\
\hline Myrceugenia ovata (H. et A.) Berg. & Myrtaceae & Huillipeta & Far & A \\
\hline Nothofagus glauca (Phil.) Krasser & Fagaceae & Hualo & $\mathrm{Fa}$ & A \\
\hline Nothofagus obliqua (Mirb.) Oerst. & Fagaceae & Roble & $\mathrm{Fa}$ & A \\
\hline Nothofagus macrocarpa (DC) F.M. Vázquez \& R. A. Rodr. & Fagaceae & Roble de Santiago & $\mathrm{Fa}$ & A \\
\hline Osmorhiza chilensis $\mathrm{H}$. et A. & Apiaceae & Apio del monte & $\mathrm{H}$ & A \\
\hline Oxalis rosea Jacq. & Oxalidaceae & Vinagrillo & $\mathrm{T}$ & A \\
\hline Perezia carthamoides $\mathrm{H}$. et A. & Cichoriaceae & Estrella de cordillera & $\mathrm{H}$ & A \\
\hline Peuтиs boldus Mol. & Monimiaceae & Boldo & $\mathrm{Fa}$ & A \\
\hline Plantago lanceolata $\mathrm{L}$. & Plantaginaceae & Siete venas & $\mathrm{H}$ & I \\
\hline Plantago major $\mathrm{L}$. & Plantaginaceae & Llantén & $\mathrm{H}$ & I \\
\hline Prunella vulgaris $\mathrm{L}$. & Lamiaceae & Hierba mora & $\mathrm{C}$ & I \\
\hline Proustia pyrifolia DC & Asteraceae & Tola blanca & $\mathrm{Ft}$ & A \\
\hline Quillaja saponaria Mol. & Rosaceae & Quillay & $\mathrm{Fa}$ & A \\
\hline Ranunculus muricatus $\mathrm{L}$. & Ranunculaceae & Hierba del guante & $\mathrm{T}$ & I \\
\hline Retanilla ephedra (Vent.) Brongn. & Rhamnaceae & Retamilla & Far & A \\
\hline Rhaphithamnus spinosus (A. L. Juss.) Mold. & Verbenaceae & Espino negro & Far & A \\
\hline Ribes punctatum $\mathrm{R}$. et $\mathrm{P}$. & Saxifragaceae & Zarzaparrilla & Far & A \\
\hline Rubus ulmifolius Schott. & Rosaceae & Zarzamora & $\mathrm{Ft}$ & I \\
\hline Rumex acetosella $\mathrm{L}$. & Polygonaceae & Romacilla & $\mathrm{H}$ & I \\
\hline Rumex conglomeratus Murray & Polygonaceae & Romaza & $\mathrm{H}$ & I \\
\hline Sanicula crassicaulis Poepp. ex DC. & Apiaceae & Huanaca & $\mathrm{H}$ & A \\
\hline
\end{tabular}


BOSQUE 25(1): 69-85, 2004

Comparación fitosociológica de los bosques de Belloto (Beilschmiedia, Lauraceae) en Chile central

\begin{tabular}{|c|c|c|c|c|}
\hline Especie & Familia & Nombre común & F & $\mathrm{O}$ \\
\hline Schinus latifolia (Gill. ex Lindl.) Engler & Anacardiaceae & Huingán & $\mathrm{Fa}$ & A \\
\hline Schinus patagonica (Phil.) Johnst. & Anacardiaceae & Huingán & Far & A \\
\hline Senecio sp. & Asteraceae & & Far & A \\
\hline Senna candolleana (Vogel) Irw. Et Barneby & Caesalpiniaceae & Quebracho & Far & A \\
\hline Sicyos bryoniifolius Moris & Cucurbitaceae & Calabacillo & $\mathrm{T}$ & I \\
\hline Silybum marianum (L.) Gaertn. & Asteraceae & Cardo Mariano & $\mathrm{T}$ & I \\
\hline Solanum maglia Schlecht. & Solanaceae & Papa silvestre & $\mathrm{Cr}$ & A \\
\hline Solanum nigrum $\mathrm{L}$. & Solanaceae & Tomatillo & $\mathrm{T}$ & I \\
\hline Sonchus asper (L.) J. Hill. & Asteraceae & Nilhue & $\mathrm{T}$ & I \\
\hline Sophora macrocarpa J. E. Sm. & Fabaceae & Mayú & Far & A \\
\hline Stachys grandidentata Lindl. & Lamiaceae & Menta & $\mathrm{C}$ & A \\
\hline Stellaria media (L.) Vill. & Caryophyllaceae & Quilloi-Quilloi & $\mathrm{T}$ & I \\
\hline Taraxacum officinale Weber & Asteraceae & Diente de león & $\mathrm{H}$ & I \\
\hline Torilis nodosa (L.) Gaertn. & Apiaceae & No conocido & $\mathrm{T}$ & I \\
\hline Tristerix tetrandrus (R. et P.) Mart. & Loranthaceae & Quintral & $\mathrm{Fp}$ & A \\
\hline Trevoa trinervis Miers. & Rhamnaceae & Tebo & Far & A \\
\hline Urtica dioica $\mathrm{L}$ & Urticaceae & Ortiga & $\mathrm{T}$ & I \\
\hline Verbascum virgatum Stockes & Scrophulariaceae & Hierba del paño & $\mathrm{T}$ & I \\
\hline Veronica anagallis-aquatica $\mathrm{L}$. & Scrophulariacae & No me olvides del campo & Cre & I \\
\hline Viviania marifolia Cav. & Vivianiaceae & Oreganillo & $\mathrm{C}$ & A \\
\hline LILIOPSIDA (Monocotiledóneas) & & & & \\
\hline Aira caryophyllea $\mathrm{L}$. & Poaceae & Aira & $\mathrm{T}$ & I \\
\hline Alstroemeria haemantha $\mathrm{R}$. et $\mathrm{P}$. & Amaryllidaceae & Amancay rojo & $\mathrm{Crg}$ & A \\
\hline Bomarea salsilla (L.) Herb. & Amaryllidaceae & Copihuillo & $\mathrm{Crt}$ & A \\
\hline Carex sp. & Cyperaceae & Cortadera & $\mathrm{H}$ & A \\
\hline Chusquea culeou Desv. & Poaceae & Colihue & $\mathrm{F}$ & A \\
\hline Chusquea cumingii Nees. & Poaceae & Colihuito & Far & A \\
\hline Chloraea chrysantha Lindl. & Orchidaceae & Orquídea & $\mathrm{Crg}$ & A \\
\hline Cynosurus echinatus L. & Poaceae & Cola de ratón & $\mathrm{T}$ & I \\
\hline Cyperus eragrostis Lam. & Cyperaceae & Cortadera & Hр & A \\
\hline Dioscorea bryoniifolia Poepp. & Dioscoreaceae & Papa cimarrona & $\mathrm{Crt}$ & A \\
\hline Dioscorea reticulata Gay & Dioscoreaceae & Papa cimarrona & $\mathrm{Crt}$ & A \\
\hline Gilliesia graminea Lindl. & Liliaceae & No conocido & $\mathrm{Crg}$ & A \\
\hline Holcus lanatus $\mathrm{L}$. & Poaceae & Pasto miel & $\mathrm{H}$ & I \\
\hline Lemna minor $\mathrm{L}$. & Lemnaceae & Lenteja de agua & $\mathrm{Crf}$ & A \\
\hline Libertia sessiliflora (Poepp.) Skottsb. & Iridaceae & Calle-Calle azul & $\mathrm{H}$ & A \\
\hline Nothoscordum striatellum (Lindl.) Kunth. & Liliaceae & Huilli de perro & $\mathrm{Crg}$ & A \\
\hline Paspalum distichum $\mathrm{L}$. & Poaceae & Chépica rastrera & $\mathrm{H}$ & I \\
\hline Poa аппиа $\mathrm{L}$. & Poaceae & Pasto piojillo & $\mathrm{T}$ & I \\
\hline Puya chilensis Mol. & Bromeliaceae & Chagual & $\mathrm{H}$ & A \\
\hline Scirpus cernuus Vahl. & Cyperaceae & Can-Can & $\mathrm{Hp}$ & A \\
\hline
\end{tabular}

Formas de vida: $\mathrm{Fa}=$ fanerófito arbóreo, $\mathrm{Far}=$ fanerófito arbustivo, $\mathrm{Fp}=$ fanerófito parásito, $\mathrm{Ft}=$ fanerófito trepador, $\mathrm{C}=$ caméfito (subarbusto), $\mathrm{H}=$ hemicriptófito (hierba perenne), $\mathrm{Hp}=$ hemicriptófito palustre, $\mathrm{Cr}=$ criptófito, $\mathrm{Crg}=$ criptófito geófito, $\mathrm{Crf}=$ criptófito acuático flotante, $\mathrm{Crn}=$ criptófito acuático natante, $\mathrm{Cre}=$ criptófito helofítico, $\mathrm{Crt}=$ criptófito trepador, $\mathrm{T}=$ terófito (hierba anual),

Origen fitogeográfico: $\mathrm{A}$ = autóctono, $\mathrm{I}$ = Alóctono. 